CBLC (Achenbach, 1991) cutoff point for clinically significant pathology was 65 of a possible 113 behavioral problem items (eg Externalizing, Internalizing; aggression, depression, hyperactivity). K-SADS diagnoses were grouped as Affective/anxiety, Disruptive, Comorbid, and symptomatic (delusions, hallucinations, social withdrawal, suicidal, irritability).

Psychopathology was identified by the CBLC and K-SADS in $26 \%$ and 51\%, respectively, of the CPS and PGE patients. Compared to the nonepileptic group, children with epilepsy had significantly higher mean CBCL scores, and more diagnoses and symptoms of psychopathology. The scores and rates of pathology did not differ in the CPS and PGE groups. IQ scores were correlated with mean CBCL scores and K-SADS diagnosis in both CPS and GPE groups, and the negative association was statistically significant in CPS patients for mean total and Externalizing CBCL scores and for irritability on the K-SADS. Poor seizure control was significantly correlated with mean total and Internalizing CBCL scores but not with K-SADS diagnoses or symptoms. The K-SADS is a more sensitive test of psychopathology than the CBCL in children with CPS and PGE. (Ott D, Caplan R, Guthrie D et al. Measures of psychopathology in children with complex partial seizures and primary generalized epilepsy with absence. I Am Acad Child Adolesc Psychiatry August 2001;40:907-914). (Reprints: Dr Derek Ott, Neuropsychiatric Institute, Room 58-242b, 760 Westwood Plaza, Los Angeles, CA 90024).

COMMENT. Children with either complex partial or primary generalized epilepsies are at increased risk of having behavioral and affective disorders. Poor seizure control may not be associated with an increased risk of psychiatric diagnoses and symptoms, but the high rate of refractory epilepsy and effect of antiepileptic drugs on behavior limit the significance of this finding. A structured diagnostic interview (K-SADS) in addition to the child behavior checklist (CBCL) may be necessary to uncover psychopathology in children with epilepsy. Children with CPS like those with GPE have high rates of both externalizing (disruptive) and internalizing (affective/anxiety) disorders. The high rates of irritability, social withdrawal, suicidal ideation, and interictal hallucinations emphasize the need for psychological counseling and psychiatric intervention in children with epilepsy.

\title{
TERATOGENICITY OF CARBAMAZEPINE
}

The teratogenic risk after first trimester exposure to carbamazenine was determined from 210 pregnancies followed prospectively at the Israeli Teratogen Information Service, and Laboratory of Teratology, Hebrew University, Hadassah Medical School, and Ministry of Health, Jerusalem, Israel. Indications for treatment with carbamazepine included epilepsy $(80 \%)$, trigeminal neuralgia or psychiatric disorder (13\%), and were unspecified in $7 \%$ of women. Carbamazepine was the only AFD in $68 \%$, and the mean daily dose was $645+/-339 \mathrm{mg}$. Pregnancy outcome was compared with two controls, matched and general, unexposed to teratogenic agents. Mean maternal age was 29.6 years.

A twofold increase in the rate of major congenital anomalies occurred in the carbamazepine compared to the general control group (12/160 vs 18/560; relative risk 2.24). A similar trend occurred comparing the carbamazepine and matched control groups (12/160 vs 6/179; RR 2.37). Birth weight was reduced by approximately $250 \mathrm{~g}$ in carbamazepine-exposed compared to control infants. Elective terminations of pregnancy were more frequent in the carbamazepine group. The rate of major congenital anomalies was not significantly different in epileptic and nonepileptic or mono- and polytherapy groups. The congenital anomalies among carbamazepine-exposed infants showed no specific pattern. The 
rate of congenital heart defects in exposed infants was $2.9 \%$ compared to $1.6 \%$ in matched controls and $0.7 \%$ in the general control group. No cases of neural tube defect were recorded, an omission thought to reflect a sample size limitation. (Diav-Citrin O, Shechtman S, Arnon J, Ornoy A. Is carbamazepine teratogenic? A prospective controlled study of 210 pregnancies. Neurology July (2 of 2) 2001;57:321-324). (Reprints: Dr Asher Ornoy, Department of Anatomy and Cell Biology, Hebrew University and Hadassah Medical School, PO Box 12272, Jerusalem, 91120, Israel).

COMMENT. In this Israeli study, one of the largest series reported, first trimester carbamazepine (CBZ) exposure was associated with a twofold increase in the rate of major congenital anomalies and a reduced infant birth weight compared to controls. Neural tube defect, the most commonly reported teratogenic effect of CBZ, was surprisingly absent. (See Progress in Pediatric Neurology I and II. PNB Publishers, 1991(pp112-113) and 1994 (pp109-112), for previous reports of AED teratogenicity, including spina bifida and $C B Z$ ). The 10,11-epoxide intermediate metabolite of $\mathrm{CBZ}$ has been implicated as the teratogenic agent. Oxcarbazepine (Trileptal ${ }^{(\mathbb{R})}$ ), the recently introduced congener of CBZ, has no epoxide metabolite, but undergoes reduction to form 10-monohydroxy derivative (MHD). Trileptal is reported to have no serious side effects and hopefully, this will include no teratogenicity.

\section{INTRACRANIAL HYPERTENSION}

\section{IDIOPATHIC INTRACRANIAL HYPERTENSION}

The diagnosis and treatment of 32 patients diagnosed with idiopathic intracranial hypertension were analysed in a retrospective chart review at the Hospital for Sick Children, Toronto, ON, Canada, and Great Ormond Street Hospital for Children, Institute of Child Health, London, UK. Ages ranged from 2 to 17.5 years. Twenty three were female. The most common presenting symptom was headache (91\%), followed by nausea and vomiting (56\%), double vision (38\%), and visual loss or blurring (25\%). Signs at presentation included papilledema (97\%), VIth cranial nerve palsy ( $31 \%$ unilateral, $9 \%$ bilateral), retinal hemorrhages (13\%), constricted visual fields (12\%), and decreased visual acuity (29\%). Associated disorders in $59 \%$ included obesity in $48 \%$, recent or recurrent otitis media (28\%), upper respiratory tract infection (16\%), sinusitis (13\%). Addison's disease and thyroiditis in 1 patient, and sudden withdrawal of cyproterone acetate for precocious puberty in 1. CT or MRI excluded hydrocephalus or spaceoccupying lesion, and none had sinus venous thrombosis. All but one had a CSF pressure of $>20 \mathrm{~cm}$ (a normal opening pressure does not negate the diagnosis, since pressure is known to rise intermittently or infrequently). None developed tonsillar herniation. Treatments intended to alleviate symptoms and prevent visual loss included acetazolamide as first line treatment (44\%), corticosteroids (short course) used for deteriorating visual loss or persistent headache (34\%), lumboperitoneal shunt (25\%), optic nerve fenestration (16\%), and repeat lumbar puncture (25\%). Combination treatments were used in $40 \%$. Four (13\%) received no treatment. Four recovered after the first lumbar puncture and never required further therapy. None of the therapies has been proven effective by randomized controlled trial. The terms "benign" and "pseudotumor" should be discarded in favor of "idiopathic intracranial hypertension." (Salman MS, Kirkham FJ, MacGregor DL. Idiopathic "benign" intracranial hypertension: case series and review. I Child Neurol July 2001;16:465-470). (Respond: Dr Michael S Salman, Division of Neurology, The Hospital for Sick Children, 555 University Avenue, Toronto, ON M5G 1Xs 